\title{
Index of works and passages
}

Note: Numbers in brackets denote sections or line numbers in within works.

Anacreontea (33) 24

Apollonius of Rhodes, Argonautica 37

Appian, Civil Wars (5.27) 41n2

Bacon, Sir Francis, De Sapientia Veterum $48 \mathrm{n} 18$

Bathurst, Theodore, Calendarium Pastorale 88n11, $127 \mathrm{n} 76$

Bion, Lament for Adonis 22, 27, 70, 107, 184-5, 213-14

fr. 13 ('Love and the Birdcatcher') 29

[Bryskett, Lodowick], 'The Mourning Muse of Thestylis' 27, 207, 277n79

Callimachus, Aitia 12, 19

'Hymn to Apollo' (105-12)

$12 \mathrm{n} 33,19,20$

Epigram (2) 209

Elyot, Sir Thomas, The Boke Named the Gouernour 4

Hesiod

Theogony (1-34) 13, 200, 278
Works and Days 13, 200

Homer

Iliad

(2.488-90) 249n44

(7.299-305) 58

(18.483-607) 18

Odyssey 19

(6.149-52) 125

(9.231-542) 249, 250-1

(11) 30

Horace, Carmina

(1.12.9-10) 280

(3.5.1-4) 178

(3.30.10-16) 240, 290

Lodge, Thomas Scyllaes Metamorphosis 28, 252n52

Lucretius, De rerum natura

(1.1-37) 292-3

(1.248-64) 293

(2.327-8) 284-5

(3.26-30) 285

(3.894-6) 294

(4.580-9) 282-4

(4.1037-287) 293-4

(5.8) 285

(5.1379-98) 283-4, 286

Lyfe of Virgil (London, 1550) 61n39 
Minturno, Antonio De poeta 18

Moschus, 'Love the Runaway' 23, 29 fr. 3 ('Alpheus') 239, 241

[Moschus], Lament for Bion 22, 107, 287, 184-5, 213-17

(1) 241-2

(44-5) 100n28, 284n85, 287

(53-4) 209, 216

(70-84) 21, 201, 239-40, 299

(93-7) 215

(99-104) 139n86

(123-4) 287, 289

Mulcaster, Richard, The passage of our most drad Soueraigne 116-23, 128, 130,142

Orosius, Seven Books of Histories Against the Pagans (6.1) 73

Ovid

Amores

(1.15) 209

(3.9) 209, 214-15

Ars amatoria (1.518) 249

Fasti (1.27-44) 147n98

(4.865-70) 130-1

(6.703-8) 104

Heroides 27

Metamorphoses 29, 277

(1.94-6) 249

(1.131-4) 259

(5.346-53) 250

(5.638) 249

(6.148-312) 125-6

(6.383-400) 104

(10.1-78) 35

(10.708-39) 27, 216

(11.132-5) 259

(11.146-7) 259

(11.153-71) 103-4, 258-9

(11.172-9) 258
(13.723-7) 252

(13.730-4) 252

(13.738-14.74) 28, 252

(13.742-5) 249

(13.759-60) 250

(13.764-5) 249

(13.780-8) 249, 250, 253-5

(13.810-30) 251

(13.865-6) 248-9

(13.867-9) 250

(13.882-97) 248

(14.75-7) 253

(15.843-8) 71n49

Pausanias, Description of Greece

(5.7.2-3) 239n22

(6.22.9) $248 \mathrm{n} 41$

(10.26.1) 35n81

Petrarch, Rime sparse 192

(60.3-4) 179-80

Plato, Phaedrus (245a) 34n80

Pliny, Natural History (2.93-4) 69, 152

Puttenham, George, Art of Poesy

(1.18) 9

(3.5-6) 6

(3.18) 233

(3.25) 233

Ralegh, Walter, Ocean to Cynthia 227-8, 232

'Our passions are most like to Floods and streames' 245

Sannazaro, De Partu Virginis

(3.196-236) 72

Servius, on

Aen. (1.287) 72n53

Aen. (3.20) 104

Aen. (3.424) 252n53

Aen. (3.661) 254

Aen. (4.58) 104 
Aen. (6.790) 72n53

Aen. (8.861) 72n53

Eclogues 42

Ecl. (1.27) 49

Ecl. (1.29) 48, 50

Ecl. (2.6) 22-3, 43, 55, 65, 75n59, 98,173

Ecl. (2.73) 43, 57-8, 93, 98

Ecl. (3.20) 42

Ecl. (3.71) 56, 77, 89, 98

Ecl. (4.1) 115

Ecl. (4.10) 69

Ecl. (4.12) 148

Ecl. (5.20) 70

Ecl. (5.29) 71

Ecl. (5.65) 144-5

Ecl. (8.6) 13 n36

Ecl. (8.55) 279

Ecl. (9.5) 58-60

Ecl. (9.23) 75

Ecl. (9.48) 148-9

Ecl. (9.50) 76

Ecl. (10.1) 91, 111, 185, 236, 239

Ecl. (10.4) 239

Ecl. (10.9) 198-9

Ecl. (10.10) 236

Ecl. (10.28) 91, 257n63

Ecl. (10.44) 264

Ecl. (10.45) 263

Ecl. (10.46) 91-2, 109, 185, 204, 214,242

Ecl. (10.71) 13

Georgics 20

Shakespeare, William, Venus and Adonis 27, 216

Sidney, Philip

Apology for Poetry 80, 196

Arcadia 187

Astrophil and Stella 187, 192-5, 208, 210n32, 214

(Song 1) 192n12

(Song 2) 192

(sonnet 24) 195 (sonnet 25) 192

(sonnet 35) 195

(sonnet 52) 192

(sonnet 62) 210

(sonnet 69) 210

(sonnet 74) 194

(sonnet 81) 194

Spenser, Edmund

Amoretti and Epithalamion

23-4, 192-3, 218-19, 221, 244

Amoretti

(65) 218

(68) 218

(75) $127 \mathrm{n} 75$

Anacreontics (4) 24, 29

Epithalamion 169, 288

(16) 35-6, 288-9

(417-23) 218-19

'Astrophel' 24, 27, 39, 183-220, 222-3, 226, 230-1, 236

prologue 203, 265

(1-6) 187, 200

(9-12) 193

(25-30) 188, 208

(31-4) 184, 189-90, 201

(37-42) 189-90, 201

(43-8) 189-90, 201

(49-54) 190-2

(61-6) 191, 195

(67-72) 195, 238

(73-8) 196

(79-80) 193

(86-8) 196

(91) 196

(101) 193

(111-12) 196

(127-32) 197

(149-50) 184

(205-6) 204

'Lay of Clorinda'

(1-6) 203-4, 219

(7-8) 204 


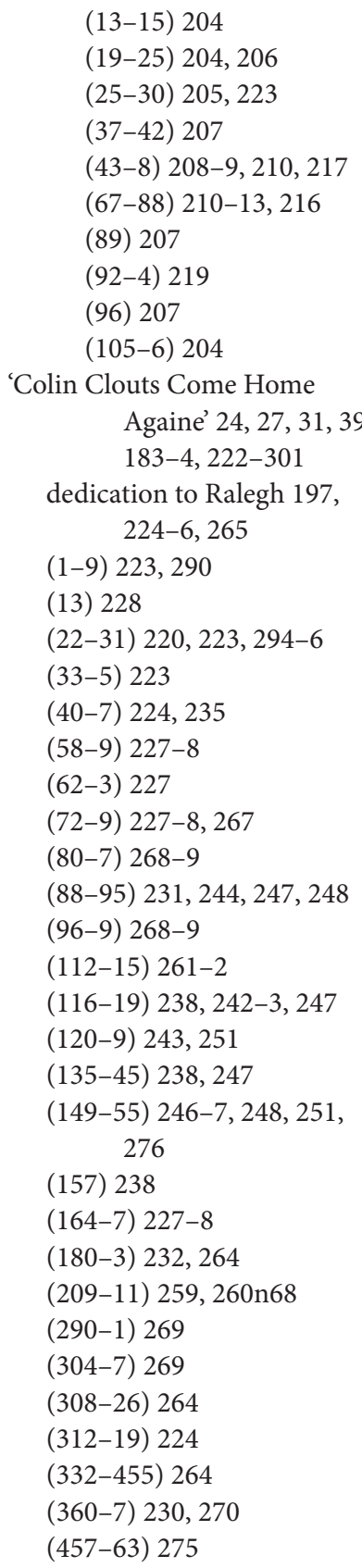

(472-9) 191, 244, 276

(480-4) 273, 275, 291

(485-615) 264

(604) 235

(612-15) 235

(616-19) 268, 274, 291

(620-7) 270, 272

(628-47) 271-2, 291-2

(654) 193

(665-6) 193

(690-8) 188, 234

(699-702) 233, 236

(703-30) 264

(707-10) 269

(726-8) 188, 243

(736) 142

(762-3) 188

(776-92) 194, 233

(789-90) 188, 195, 233

(799-804) 212, 237

(823-38) 212, 237, 269, 272

(839-42) 218, 237

(863-72) 237

(870-1) 218

(888) 218

(891-4) 232, 233, 264

(899-902) 272

(903-6) 232, 273

(907-26) 273-4

(927-9) 276

(947-51) 276

(952-5) 276-7

'Mother Hubberds Tale' 30,

$$
31-3,243
$$

'Muiopotmos' 29, 30

'Ruines of Time'

(4-7) 262

(332-6) 201

(390-2) 35

The Faerie Queene

(I.Pr.1) 10, 16, 23, 182

(I.Pr.3) 293

(I.i.23) 30n73 
(I.i.35) 234

(I.ii) 23

(I.viii) 208

(II.vii.29) 235-6

(II.vii.44-50) 234-6

(III) 244

(III.Pr.4) 228

(III.i.25) 243

(III.ii) 29

(III.v.42-7) 228, 245

(III.vi.11-23) 29

(III.vi.29-50) 23, 245, 293

(III.vi.30-3) 211, 213

(III.vi.34) 218

(III.vi.41-2) 211

(III.vi.47) 216

(III.vi.48) 211-12

(IV.ii.1) 37

(IV.vii.35-viii.17) 228-9, 245

(IV.x.44-7) 293

(IV.x.58) 35-6

(V.Pr.5-6) 151-2

(V.ix.25-6) 33, 247

(VI) 25

(VI.ix.24-5) 224

(VI.x.10-28) 278-9

(VII) 29

(VII.vi.38-55) 29, 33, 248n41

Dedicatory Sonnet 14 (to Walter Ralegh) 230-1

The Shepheardes Calender

'Epistle to Harvey' 8-9, 16, $37,149,181$

'Generall argument' 147, 156, 162

Januarye 84-93, 128, 134,

$160,163,166,170,174$

Argument 85, 165

(5-6) 85

(13-18) 90-1

(19-20) 94

(39-40) 88, 142, 165

(43-8) 85
(49-54) 86, 129, 223

(55-60) 88, 143-4, 192

(63-6) 89, 104

(67-72) 90, 140

emblem 93, 106

(1 gloss) 9

(57 gloss) 88

(59 gloss) 88-9

(60 gloss) 86, 126

emblem gloss 106

Februarie 163, 170-1

March 29, 163, 167, 168, 173

(40-2) 163

(100) 163

Aprill 38, 114-35, 136, 160, $163,166-7,170$, 235-6

Argument 98, 114, 129, 141

$(9-12) 129$

(13-16) 128-9

(25) 232

(37-41) 97, 130

(43-4) 111

(48) $111,116,130,139$

(50-1) 97, 121

(55-63) 116, 120, 130,

164

(64-5) 97, 130

(73-8) 156

(82-5) 97,130

(86-90) 125-6, 274

(91-4) 97, 121

(95-9) 124, 127, 143

(100-3) 97,100

(104) 116,139

(109-17) 97, 127

(122-6) 116, 139

(127-35) 130, 131, 140

(136-44) 116, 120, 131, $139,164,189-90$

(147-53) 127-8, 129, 144

(154-7) 131, 232 


\begin{tabular}{|c|c|}
\hline (158-61) 108, 132, 232 & $(145-6) 173$ \\
\hline emblems 125,130 & (151-89) 204, 288 \\
\hline (26 gloss) $126-7,129,140$ & $(190-3) 174,201$ \\
\hline 274 & (131 gloss) 173 \\
\hline (50 gloss) $121,134,141$ & September 164, 166 \\
\hline 155 & (91) 243-4 \\
\hline (68 gloss) 121 & $(136-9) 67$ \\
\hline (86-7 gloss) 125 & (177) 174 \\
\hline Maye $163-4,166$ & $(254-7) 67$ \\
\hline (14) 164 & October $39,163,164,168$ \\
\hline June $93-114,160,163,164$, & $171-3$ \\
\hline $167-8,170,174-6,231$ & Argument 176, 179, 270 \\
\hline$(1-8) 94,99$ & (25-30) $287-8,291$ \\
\hline$(9-13) 95,98,111$ & $(37-40) 9,16,35$ \\
\hline$(14-16) 96,113$ & (55-66) $172-3,177$ \\
\hline$(17-24) 96,99$ & $(83-4) 177,232 \mathrm{n} 13$ \\
\hline$(25-32) 96,100$ & (93-4) 179,270 \\
\hline$(33-8) 97,98$ & $(96-8) 179$ \\
\hline$(41-6) 97-9,140,143$ & (109-18) 172 \\
\hline $189-90$ & (1 gloss) $9,173-4$ \\
\hline (49-56) 99-100, 109, 140, & (21 gloss) 176 \\
\hline $189-90,287$ & (27 gloss) $165-6,177,288$ \\
\hline (57-64) 100-2, 189-90 & (28 gloss) 35,37 \\
\hline$(65-72) 102-5,141$ & (65 gloss) $176-7$ \\
\hline 259 n66 & (93 gloss) $179-80$ \\
\hline$(73-80) 103,141$ & (113 gloss) 172 \\
\hline$(81-2) 105$ & Nouember $38,114,135-58$ \\
\hline$(91-6) 107,287$ & $160,164-6,167,168$ \\
\hline$(100-4) 108,110,113,156$ & $170,183,202,222,295$ \\
\hline 158 & Argument 138, 140 \\
\hline$(106-12) 110,111,129$ & $(7-8) 136$ \\
\hline $131,156,272$ & (9-17) 136-7, 150-2, 157, \\
\hline$(113-20) 111,174$ & 165 \\
\hline emblem 106 & (30) 201 \\
\hline (25 gloss) $157-8$ & $(31-2) 137$ \\
\hline (43 gloss) 98 & (43-4) 141 \\
\hline (81 gloss) 105 & $(50-1) 141$ \\
\hline (97 gloss) 108 & $(67-8) 137,156$ \\
\hline (102 gloss) 108 & (71) 189-90 \\
\hline Julye 164,166 & $(75-6) 139,206$ \\
\hline August $160,163,164,166-7$, & (77-9) $140,208-9$ \\
\hline 168,170 & (83-9) 139, 206 \\
\hline (101) 163 & (93-102) 143-5 \\
\hline
\end{tabular}




$$
\begin{aligned}
& (108-9) 139,140,165, \\
& 207 \\
& (113-22) 141-2,189-90 \\
& (124-9) 137 \\
& (133-5) 137 \\
& (136-7) 154 \\
& (143-7) 138,207 \\
& (153-7) 142-3 \\
& (166-9) 153 \\
& (171-2) 140 \\
& (175-6) 153-6 \\
& (178-82) 153,155,158 \\
& (185-8) 154 \\
& (191) 154-5,158 \\
& (193-9) 154 \\
& (16 \text { gloss }) 141,150 \\
& (38 \text { gloss } 138,140-1
\end{aligned}
$$

December 147, 160, 163, $164-5,166,170,182$$$
\text { (45-6) } 201
$$$$
\text { (91) } 174
$$$$
\text { (143-4) } 157
$$

Viewe of the Present State of Ireland 266

'Virgils Gnat' 30-1, 35, 289

Theocritus, Idylls 17
(1) $22,104,135,161,165,166$, 184-5
(1.1-14) 284
(1.15-18) 136
(1.20) 18
(1.29-55) 18-19
(1.66-9) 197
(1.81-7) 190
(1.117) 201, 239
(1.140) 197-8
(1.141) 105
(2) 59,60 n 35
(3) $18,22,52,88$
(3.1-7) 45-6, 53, 75, 133
(3.10-11) 54,55
(3.22-3) 54

$$
\begin{aligned}
& (3.25-6) 46 \\
& (3.34-6) 53 \\
& (3.52) 90 \\
& (5) 18 \\
& (7) 22,73 \\
& (7.21-22) 55 \\
& (7.36) 18 \\
& (7.43-4) 278 \\
& (7.47-9) 18,19 \\
& (7.71-8) 45,106-7,185,205 \\
& (7.87-9) 52 \\
& (7.96-7) 126 \\
& (7.133-47) 51-2,284 \\
& (11) 47-8,53,57,75,88,90,134, \\
& \quad 249,251,253-4 \\
& (11.1-4) 22 \\
& (11.50-3) 250 \\
& (13) 18,19 \\
& (15) 18,213 \\
& (16) 18 \\
& (17) 18,41 \mathrm{n} 1 \\
& (17.1-4) 177-8,232 \mathrm{n} 13 \\
& (24) 18,19 \\
& \text { (1) } 19
\end{aligned}
$$

[Theocritus] (19) 24, 29

Virgil, Aeneid

(1.1a-d) (disputed Ille ego opening) 10,182

(1.1) 23

(1.1-7) 96, 113

(1.201-2) 252

(1.328) 125

(2.199-227) 30

(2.740-95) 33-4

(3.420-8) 252

(3.559) 253

(3.565) 260n 68

(3.570-82) 250, 251-2

(3.614-15) 257n64

(3.619-20) 251

(3.632) 251

(3.655-61) 252, 254-5

(3.664) 251 
(3.678) 251

(3.670-4) 251, 254, 255-6

(3.683-5) 253

(3.690-1) 252

(3.694-6) 239-40, 253

(4) $23,32,146$

(6) 23,30

(6.472-4) 158

$(6.748-51) 213$

(6.847-53) 35

(8) 25

(12.952) 25

Eclogues

(1) $16,26,41,43,79,84-8$, $105,134,161,170$

(1.1-5) 15, 25, 45-6, 52, 64, $66,74,94,95,99-100$, 108-9, 112, 180, 228, $281,284,287$

(1.6-8) 15, 27, 41n1, 71, 124, 235

(1.9-10) 95, 127

(1.12-13) 85

(1.16-17) 66

(1.27-39) 46-50

(1.31-5) 87-8, 93, 106

(1.36-9) 220, 223

(1.42) 161

(1.45) 48

(1.46-50) 95

(1.51-8) 94-5

(1.59-63) 62-4, 72, 224, 235, 247n 40

(1.64-6) 95-6

(1.67-9) 62

$(1.70-2) 61$

(1.73) 49, 76, 147

(1.74-8) 62, 90

(1.79-84) 25, 67

(2) $22,43,75,88,133-4,161$, 170-1

(2.2) 89

(2.5) 65
(2.6-7) $22-3,53,59,89$

(2.8-11) 54, 59n 35

(2.12-13) 54

(2.14-15) 54

(2.32-3) 91, 283

(2.36-7) 167n10, 249

(2.40-4) 53, 56-7, 88

(2.45-50) 54, 56n 31,143

(2.51-2) 54, 88, 98

(2.56) 88,142

(2.69-73) 57, 88-9, 93, 174,

242

(3) 161,173

(3.33-4) 163

(3.55-7) 284

(3.60) 177

(3.70-71) 54, 55, 77, 90

(3.94-7) 74

(3.109) 173

(3.110) 241

(4) $26,38,43,77,79,114,122$, $128,130,161,166,175$

(4.1-3) 11, 12-13, 15, 102-3, $115,269,300$

(4.6) $69,123-4$

(4.10) 69

(4.17) 69

(4.31-9) 260

(4.53-9) 11, 26, 27, 33, 101, 200, 201, 215, 262, 281, 300

(5) $11,26,38,43,79,104,114$, $135,146-9,152-3,184$, 202, 222, 296-7

structural centre of Eclogues 161, 163-5, 296

(5.10-15) 136, 229

(5.16-18) 137

$(5.20-3) 70,74,76,223$

(5.24-8) 137, 223

(5.34-9) 137, 205, 223

$(5.38-9) 74$

$(5.40-1) 74$ 


\begin{tabular}{|c|c|}
\hline (5.43) 70, 291 & $(9.5-6) 58-60$ \\
\hline$(5.45-7) 286$ & $(9.7-13) 33,63-4,105-6,132$ \\
\hline (5.51-2) 70, 75, 135, 291 & $(9.11-13) 33,132$ \\
\hline (5.56-80) 134,155 & $(9.14-16) 106$ \\
\hline (5.56-8) 285 & (9.19-20) 74 \\
\hline$(5.62-4) 33,70,72,73,205$ & $(9.23-5) 74-5,133$ \\
\hline $221,223,284-5$ & $(9.26-9) 26,56,75,101,134$, \\
\hline (5.65-73) 144, 147, 149, 154, & $146,153,178,277-8$ \\
\hline 295 & $295-6$ \\
\hline (5.76-80) 72,73 & $(9.39-43) 75$ \\
\hline (5.86-7) 73, 75 & $(9.46-50) 76,134,146-7$ \\
\hline (5.88-90) 278 & $153-5$ \\
\hline (6) $161,174-5$ & $(9.51-4) 77,133$ \\
\hline$(6.1-2) 17,112,240 \mathrm{n} 25$ & (9.67) 77 \\
\hline (6.3-5) 11-12, 15, 16, & (10) $22,24,26,34,39,104$, \\
\hline $112-13,124,268$ & $147,164-5,182-7$ \\
\hline (6.6-8) 112, 278, 284 & $221,222,228,231$ \\
\hline$(6.29-30) 280,281$ & $236,296-7$ \\
\hline$(6.64-73) 13,33,39,187-8$, & (10.1-3) 109-11, 186, 198, \\
\hline 199-201, 205, 215, & $200,204,225,230$ \\
\hline 231,278 & 238,245 \\
\hline$(6.84-6) 175-6 \mathrm{n} 20,277,284$ & (10.4-6) 238-42, 245-6 \\
\hline $285,295-6$ & (10.8) 109, 204 \\
\hline (7) 161,173 & (10.9-12) 197-202 \\
\hline$(7.1-13) 187,262,284$ & (10.13-15) 107, 205 \\
\hline$(7.53-60) 220$ & $(10.17-18) 214$ \\
\hline (7.70) 174 & $(10.22-3) 108$ \\
\hline (8) $22,26,133-4,161$ & $(10.24-6) 284$ \\
\hline$(8.1-5) 13,33,279-80,288$, & $(10.28-30) 90$ \\
\hline 291 & $(10.31-4) 110,185-6,204$ \\
\hline$(8.6-13) 13,26,27,112$ & 208,271 \\
\hline $171-2,177,232 \mathrm{n} 13$ & $(10.44-8) 263-4$ \\
\hline$(8.22-4) 280$ & $(10.50-4) 186,200,228,229$, \\
\hline (8.33) 89 & 271 \\
\hline (8.52-56) 33, 63, 279 & $(10.60-3) 90,186,200$ \\
\hline$(8.58-60) 63,198$ & $(10.70-2) 13,182,201,215$, \\
\hline$(8.69-71) 59,290$ & 225,242 \\
\hline (8.95-99) 59-60 & $(10.73-4) 186,228,229$ \\
\hline (8.109) 59 & $(10.75-6) 13,15,16,180$ \\
\hline (9) $16,26,27,38,41,43$, & $182,265,277$ \\
\hline $73-7,105-6,135-6$ & (10.77) 111,182 \\
\hline $146-7,161,170$ & Georgics 200 \\
\hline$(9.2-3) 62$ & (3.8-9) $14,15,16$ \\
\hline
\end{tabular}




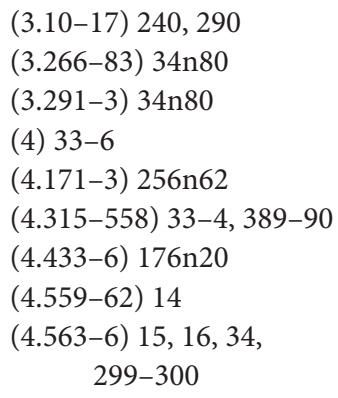

[Virgil]

Ciris 29

Culex 7, 30, 31, 35, 289

Dirae 26, 48n19, 60-8, 178, 265
(4-8) $62-5$
$(18-19) 65$
(26-36) 61, 65-7
$(48-62) 63$
(80-3) 61-2
(86-94) 62
$(98-101) 62$ 